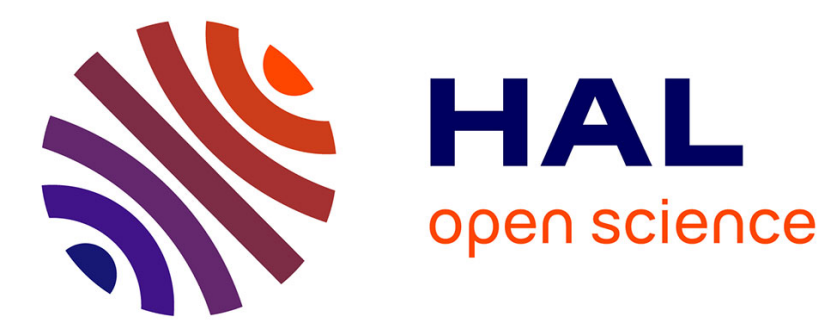

\title{
Stérilités mâle et femelle dans le genre Vitis. II. Conséquences en génétique et sélection
}

Alain Carbonneau

\section{To cite this version:}

Alain Carbonneau. Stérilités mâle et femelle dans le genre Vitis. II. Conséquences en génétique et sélection. Agronomie, 1983, 3 (7), pp.645-649. hal-00884554

\section{HAL Id: hal-00884554 https://hal.science/hal-00884554}

Submitted on 1 Jan 1983

HAL is a multi-disciplinary open access archive for the deposit and dissemination of scientific research documents, whether they are published or not. The documents may come from teaching and research institutions in France or abroad, or from public or private research centers.
L'archive ouverte pluridisciplinaire HAL, est destinée au dépôt et à la diffusion de documents scientifiques de niveau recherche, publiés ou non, émanant des établissements d'enseignement et de recherche français ou étrangers, des laboratoires publics ou privés. 


\title{
Stérilités mâle et femelle dans le genre Vitis. II. Consé- quences en génétique et sélection
}

\author{
Alain CARBONNEAU \\ I.N.R.A., Station de Recherches de Viticulture, Centre de Recherches de Bordeaux, F 33140 Pont-de-la-Maye
}

RÉSUMÉ

Les conséquences d'un nouveau modèle de l'hérédité des stérilités mâle et femelle dans le genre Vitis sont discutées sur :

- certains aspects de biologie moléculaire,

- le choix de géniteurs particuliers,

- l'évolution de la vigne,

- les stratégies de sélection.

Mots clés additionnels : Vigne, multiallélisme, épistasie, gène suppresseur, évolution, stratégie de sélection.

The consequences of a new model of the inheritance of male and female sterility in the genus Vitis are discussed in relation to: 1) some aspects of molecular biology ; 2) the choice of particular parents ; 3) the evolution of grapevine; 4) breeding strategies.

Additional key words : Grapevine, multiallelism, epistasy, suppressor gene, evolution, breeding strategy.

\section{INTRODUCTION}

Un nouveau modèle de l'hérédité des stérilités mâle et femelle dans le genre Vitis a été exposé dans l'étude précédente (CARBONNEAU, 1983). Il convient d'en développer les conséquences pour améliorer le choix des géniteurs et des méthodes de sélection, ainsi que la compréhension de l'origine de la vigne et de certains mécanismes de biologic moléculaire.

\section{INTERPRÉTATION DU MODÈLE EN BIOLOGIE MOLÉCULAIRE}

L'étude précédente a porté sur des fonctions alléliques sans préjuger de la nature biochimique des phénomènes et se résume ainsi :

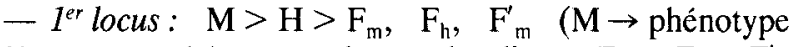
mâle ; $\mathrm{H} \rightarrow$ phénotype hermaphrodite $; \mathrm{F}_{\mathrm{m}}, \mathrm{F}_{\mathrm{h}}, \mathrm{F}_{\mathrm{m}}^{\prime}$ $\rightarrow$ phénotype femelle);

- $2^{e}$ locus : $\mathrm{E}>\mathrm{e}$; sur le même chromosome avec un taux de recombinaison voisin de 0,3 (0,5 signifiant l'indépendance totale des loci);
- avec les relations d'épistasie
$\left(E, F_{m}\right)=M, \quad(E$

- et de dosage occasionnel: $(\mathrm{E}+\mathrm{E}), \quad \mathrm{F}_{\mathrm{m}}^{\prime}=\mathrm{M}$; $(\mathrm{E}+\mathrm{e}), \mathrm{F}_{\mathrm{m}}^{\prime}=$ intermédiaire $\ll \mathrm{M}-\mathrm{H} » ;(\mathrm{e}+\mathrm{e}), \mathrm{F}_{\mathrm{m}}^{\prime}=\mathrm{F}$.

Sur le plan de la biologie moléculaire, le multiallélisme est très courant chez la plupart des espèces, notamment dans le cas des phénomènes d'incompatibilité sexuelle. Les effets épistatiques entre allèles de loci différents ont également été montrés à cette occasion (LEWIS \& JOHN, 1968). De tels effets épistatiques pourraient également trouver une explication dans le modèle de régulation proposé chez les plantes supérieures par BRITTEN \& DAVIDSON (1969).

Toutefois d'autres explications sont possibles. En particulier le modèle suivant répond tout à fait aux contraintes de fonctions résumées précédemment (il est possible de vérifier que c'est le seul capable d'expliquer les disjonctions observées dans l'hypothèse de l'intervention des 3 loci ci-dessous) :

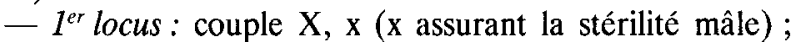

- $2^{e}$ locus : couple $\mathrm{Y}, \mathrm{y}$ ( $\mathrm{Y}$ assurant la stérilité femelle);

- transcription de l'information des 2 loci sur un même ARN messager, donc aucune recombinaison entre les 2 loci avec en plus effet polaire de la mutation $\mathrm{x}$ sur le couple (Y, y) ;

- effet suppressif sur la mutation $\mathrm{x}$ (donc mutation non sens) dû à un gène suppresseur $Z$, le locus $(Z, z)$ étant situé sur le même chromosome que le cistron $(X ; x-Y, y)$ à un taux de recombinaison de 0,3 . 
Ceci revient aux équivalences suivantes entre les 2 modèles :

$$
\begin{aligned}
& M=X-Y, \\
& H=X-y \\
& F_{m}=x-Y \\
& F_{h}=x-y \\
& E, e=Z, z
\end{aligned}
$$

Il est donc théoriquement possible dans le cas de l'hérédité des caractères sexuels chez la vigne d'avancer l'hypothèse d'une mutation non sens à effet polaire de type " cis » en liaison. avec la présence d'un gène suppresseur de cette mutation. Toutefois ce phénomène, mis en évidence chez les bactéries notamment, s'accompagne généralement d'un faible développement de l'individu dont le fonctionnement du génome, au moment de la lecture du code héréditaire, est fortement perturbé par la présence d'un suppresseur.

Une telle contrainte paraît difficilement admissible chez la vigne d'autant par exemple que les mâles issus du croisement "Counoise" $x$ "Aleatico" ne présentent aucun signe de baisse de vigueur et sont même assez remarquables pour certains d'entre eux par leur fertilité élevée (DOAZAN, comm. pers., 1982). De façon plus générale encore, le caractère mâle serait lié à une fertilité élevée comme chez les Lambrusques (CASTERAN, comm. pers., 1982) ou à des capacités de régénération intéressantes à partir de cultures d'anthères (RAIASEKARAN \& MULLINS, 1979). Le caractère mâle paraît donc souvent associé à une efficacité métabolique notoire.

Il faut donc soit rejeter cette hypothèse chez la vigne et ne retenir que les interactions épistatiques entre allèles nombreux pour un même locus, soit admettre que chez les eucaryotes existeraient des mécanismes biochimiques capables de contrôler les effets « dévastateurs" des gènes suppresseurs ce qui ouvrirait la voie d'une nouvelle conception de la génétique chez les espèces supérieures.

En tout état de cause le point important est la présence chez la vigne d'effets épistatiques au sens large du terme portant sur les caractères sexuels en liaison probable avec la sensibilité à la coulure voire avec la fertilité. Il est intéressant d'envisager les principales conséquences de ces effets épistatiques pour l'amélioration de la vigne et l'explication de son évolution.

\section{APPLICATION À L'HISTOIRE DE L'ÉVOLUTION DE LA VIGNE}

Le cas des vignes sauvages ou Lambrusques considérées par RIVES $(1962,1974,1975)$ comme les ancêtres des vignes cultivées européennes n'est pourtant pas à retenir absolument dans l'optique précédente. En effet, le caractère dioïque des Lambrusques n'est pas obligatoirement un signe d'antériorité par rapport à l'apparition des variétés monoïques cultivées, mais peut tout aussi bien, d'après la nouvelle théorie proposée, être considéré comme une manifestation postérieure à celle régissant l'apparition de ces variétés cultivées. En d'autres termes, les Lambrusques ont autant de chances d'être des ancêtres de ces variétés cultivées (Lambrusques spontanées, LEVADOUX, 1956) et des sources de variabilité intéressantes, que d'en être des descendants retournés au milieu sauvage plus sélectif, il y a peut-être fort longtemps du reste, avec beaucoup moins de possibilités de procurer une information génétique nouvelle (Lambrusques subspontanées et post-culturales, LEVADOUX, 1956). Cette interprétation se fonde sur l'apparition de mâles et de femelles dans la descendance des croisements entre certains hermaphrodites, y compris des autofécondations.

En fonction des résultats du modèle précédent et des données établies concernant les origines de la vigne (LeVADOUX, 1956 ; RIVES, 1962, 1974, 1975), il est intéressant d'essayer de classer les divers types de Lambrusques. Pour ce faire, un scénario vraisemblable de l'évolution de la vigne est envisageable ; il se décompose en 7 phases :

1) Les divers types floraux étant actuellement tous constitués à partir du même modèle de base hermaphrodite, avec pour les mâles ou les femelles une atrophie mais non une disparition du pistil ou des étamines, il est logique de supposer que les premières vignes répandues à l'ère tertiaire (à feuilles entières), ceci en milieu subtropical favorable, étaient monoïques, à autofécondation dominante et homozygotes $(\mathrm{H} / \mathrm{H})$.

2) De nombreuses mutations. ne manquèrent certaincment pas alors de se produire. Par la suite, lors de variations du milieu vers des conditions moins favorables, certaines furent sélectionnées, en même temps que fut réalisée une évolution de l'état monoïque strict vers un état plus dioïque permettant une meilleure adaptation des populations. L'hétérozygotie se développa au $1^{\text {cr }}$ locus sous la forme du couple allélique $\left(\mathrm{HF} / \mathrm{F}_{\mathrm{h}}\right), \mathrm{F}_{\mathrm{h}}$ constituant en fait la mutation «type femelle» à elle seule juste suffisante pour fairc progresser la dioécie dans les populations de départ hermaphrodites. La mutation "type mâle " ne possède en effet pas une telle capacité.

3) Des retours à des milieux plus favorables rendirent l'état monoïque de nouveau plus intéressant. Mais, face aux multiples fluctuations des conditions de milieu liées en particulier aux transgressions et régressions marines, l'allèle $\mathrm{H}$, impliquant une trop grande stabilité des génotypes, ne s'imposa plus. Il se sélectionna alors un système très souple permettant par le jeu des balances alléliques d'orienter la vigne soit vers l'état monoïque en période stable favorable, soit vers l'état dioïque lors des changements du milieu : ainsi les allèles $H$ et $F_{h}$ furent conservés, mais avec adjonction de l'allèle $\mathrm{E}$, mutation de $\mathrm{e}$, permettant le retour de la population à un état plus monoïque.

4) A l'époque quaternaire vraisemblablement, le milieu devenant franchement hostile dans les zones où la vigne s'était déjà installée, une dioécie absolue s'imposa pour maximiser l'hétérozygotie et la sélection de nouveaux et rares individus adaptés. Les allèles $M$ et $F_{m}$ se généralisèrent considérablement : $M$ imposa une dioécie assez irréversible, utile dans les zones les plus marginales, et aillcurs $\mathrm{F}_{\mathrm{m}}$ en combinaison avec $E$ permit cet état mais avec une extraordinaire souplesse liée à des possibilités de retour vers un état plus monoïque dans le cas d'une association avec $\mathrm{F}_{\mathrm{h}}$, par exemple lors des périodes interglaciaires. La pré-existence de l'allèle $\mathrm{E}$ à l'époque quaternaire permettrait d'expliquer la haute adaptabilité du genre Vitis liće à unc relative rapidité de changement des types sexuels donc des balances alléliques, au cours de cette période très contrastéc.

L'apparition des mâles à une époque plus tardive, ou du moins différente de celle concernant d'abord les hermaphrodites puis les femelles, semble confirmée par l'existence de caractères morphologiques plutôt spécifiques des mâles : l'abondance des inflorescences, facteur de l'intensité de la pollinisation donc d'un brassage génétique maximum, et la découpure prononcée des feuilles. Sur ce dernier point, il est intéressant de noter qu'une feuille très découpée permet un passage substantiel de la lumière, cet effet microclimati- 
que favorisant certainement la cohabitation avec les arbres forestiers qui, dans cette période difficile, ont plus hébergé la vigne qu'ils ne se sont fait coloniser par elle. Un détail qui va à l'appui de cette thèse concerne la morphologie de cette liane qu'est la vigne qui n'enlace ni n'étouffe les arbres mais qui s'y suspend simplement. Les mutations de découpure du limbe, notamment développées dans ces zones refuges, furent véhiculées surtout par les génotypes mâles en raison de la dominance de $\mathrm{M}$ et de $\mathrm{E}$ donc de leur prépondérance numérique dans les populations, nécessaire du reste pour imposer l'état diö̈que à ces populations largement monö̈ques. Il est à remarquer enfin qu'aucune vigne fossile du tertiaire ne présentait la moindre découpure du limbe.

5) L'arrivée des conditions actuelles plus favorables mais non idéales en comparaison du climat subtropical de l'ère tertiaire, permit aux diverses populations fragmentées dans ces zones refuges de les déborder et d'évoluer en fonction de l'intérêt de l'état monoïque toujours présent sur le plan génétique. Donc sans perdre les acquis de l'évolution qui avait juste précédé (allèles $M, F_{m}$ et $E$ nécessaires) et dans de tels milieux suffisamment favorables (allèles $\mathrm{H}, \mathrm{F}_{\mathrm{h}}, \mathrm{E}$ nécessaires), l'ensemble des allèles $\left(\mathrm{M}, \mathrm{H}, \mathrm{F}_{\mathrm{m}}, \mathrm{F}_{\mathrm{h}}, \mathrm{E}, \mathrm{e}\right)$ se maintint. Ceci s'est sans doute effectué dans des rapports alléliques variables selon les diverses situations colonisées de nouveau, en fonction des contraintes imposées par ces divers milieux au développement de la vigne. La persistance de ce multiallélisme expliquerait ce qui suit.

6) L'homme découvrit la vigne et divers usages que l'on pouvait tirer de ses fruits, sans doute à partir du néolithique. Après une phase de cueillette, il cultiva bien évidemment les hermaphrodites, avec quelques femelles cependant. La condition de cette évolution fut que l'homme put trouver à sa portée un nombre suffisant d'hermaphrodites présentant diverses caractéristiques attractives au niveau du fruit notamment, afin de le décider à en cultiver après en avoir choisi les plus dignes.

La viticulture eut donc pour conséquence d'augmenter considérablement la fréquence de l'allèle $\mathrm{H}$ le plus anciennement apparu et de renverser en fait le cours de l'évolution. La multiplication végétative et les conditions de culture créèrent un milieu artificiel voire anti-écologique, ce qui permit l'essor de génotypes très différents des Lambrusques, en particulier un retour à une homozygotie plus élevée. En effet, des cépages relativement récents comme l'« Ugni blanc», donnent, contrairement à la majorité des cépages, des descendants d'autofécondation assez vigoureux ; par ailleurs, le « Muscat de Hambourg » est homozygote $(\mathrm{H} / \mathrm{H})$. En outre, une tendance à la disparition de la découpure des feuilles, développée surtout lors de l'étape vers la dioécie, est observée : c'est l'exemple du «Merlot » apparu probablement en Aquitaine après les types « Cabernets » dont le «Cabernet-Sauvignon ».

La question est de savoir, à l'aide de quelques données fournies par le modèle, si le développement des cépages en Europe s'est effectué, soit localement à partir des diverses zones refuges à la fin des dernières glaciations, soit par introduction à partir d'un centre commun situé au MoyenOrient, probablement en Arménie. Dans cette région les populations de Lambrusques comportent une proportion appréciable d'hermaphrodites. Mais cette condition piéculturale est présente également dans d'autres situations, en particulier dans les massifs rhénans. Cette diversité dans les centres d'origine des cépages, paraît corroborée par l'existence d'un multiallélisme non équifréquent pour les types " $F_{x}$ » (exemples de la «Counoise » et du « Muscat Ottonel ») ainsi que par la présence de nombreuses variations morphologiques pour une formule sexuelle donnée.
Pour conclure, les références historiques donneraient plutôt la préférence à la $2^{\mathrm{c}}$ hypothèse, mais l'étude des Lambrusques spontanées et la toponymie de certains vieux cépages («Sauvignon», «Riesling», etc) ferait pencher vers la $1^{\text {re }}$. L'adoption d'un compromis faisant état, pour l'essentiel, de croisements entre des cépages importés du Moyen-Orient et des Lambrusques spontanées européennes et, en tout cas, d'une certaine diversité dans les centres d'origine des cépages qui semble indiquer par l'étude des allèles $« F_{x} »$, prévaut actuellement.

7) La dernière étape de cette évolution est la concentration des régions viticoles dans le monde moderne qui a abouti à l'abandon de nombreux cépages et vignobles dont l'apogée se situait sans doute à la fin du Moyen-Age après l'impulsion que l'Eglise avait insufflée à la viticulture pour ses besoins propres. De vieilles vignes sont donc retournées à l'état sauvage, en conditions plus difficiles où la dioécie est intéressante. Ainsi se sont constituées les Lambrusques postculturales et subspontanées. C'est sans doute le cas des Lambrusques du pays basque dont les types sexuels ont été rapportés par RIVES (1974). Cette prospection a fourni 37 mâles (46 p. 100), 2 hermaphrodites (2 p. 100) et 42 femelles (52 p. 100). La très faible proportion d'hermaphrodites ainsi que les faibles conditions d'isolement du lieu paraissent exclure l'hypothèse, pour ces Lambrusques, de leur qualité d'ancêtre direct des cépages du Sud-Ouest par exemple.

Par contre, une interprétation peut être avancée dans l'optique d'une provenance de ces Lambrusques de quelques cépages hermaphrodites existants ou perdus, d'origine locale ou lointaine, dont les croisements auraient pu donner des mâles $\left(\mathrm{F}_{\mathrm{m}} \mathrm{E} / \mathrm{F}_{\mathrm{h}} \mathrm{e}\right)$ et des femelles $\left(\mathrm{F}_{\mathrm{m}} \mathrm{e} / \mathrm{F}_{\mathrm{h}} \mathrm{e}\right)$; la nécessité de la dioécie aurait sélectionné ces 2 derniers types. Or le résultat du croisement entre les 2 génotypes précédents est : 42,5 p. 100 de mâles; 7,5 p. 100 d'hermaphrodites ; 50 p. 100 de femelles. La confrontation avec la proportion des sexes chez les Lambrusques du pays basque est significative.

\section{CONSÉQUENCES SUR LE CHOIX DES GÉNITEURS}

Un affinement des études génétiques des cépages est, de toute façon, indispensable pour mieux étayer les hypothèses précédentes et surtout pour choisir les géniteurs les plus aptes à induire une variabilité intéressante. Une piste est fournie par ceux qui possèdent l'allèle à effet épistatique E.

Un autre point concerne le problème de l'évolution de la découpure des feuilles. La culture in vitro régénère des variants qui présentent des caractères juvéniles et une découpure nettement plus accentuée des feuilles (GRENAN, comm. pers., 1982). Ceci peut être mis en parallèle avec la découpure très prononcée des Lambrusques, surtout des types mâles, et, à ce niveau, la culture in vitro pourrait être un révélateur intéressant de certaines potentialités inexploitées normalement par le génotype, qui peuvent en outre refléter leur origine génétique. $\mathrm{La}$ culture in vitro de cépages possédant l'allèle E, par exemple le «CabernetFranc », pourrait être uné voie de recherche. En tout cas, la vigne en général constitue un modèle d'étude de choix en raison de sa rusticité et de sa grande souplesse d'adaptation liée en partie à des originalités génétiques portant sur les caractères sexuels.

La réflexion sur le choix des géniteurs doit également se porter sur le sous-genre Muscadinia. Malgré la différence concernant le nombre chromosomique entre les sous-genres 
Muscadinia et Euvitis, il apparaît que l'hérédité des caractères sexuels est la même pour tout le genre Vitis. Les disjonctions observées par LOOMIS \& WILLIAMS (1957) chez Vitis rotundifolia sont parfaitement explicables par le modèle proposé. Ce fait tendrait à renforcer la parenté globale entre les vignes et les muscadines pour certains chromosomes et donc à donner plus de poids à l'idée que les caractères spécifiques des muscadines relatifs à la résistance aux maladies et aux arômes du fruit (BOUQUET, 1978) seraient portés par une faible partie du génome de Vitis rotundifolia, éventuellement localisable cytologiquement et recombinable en croisement.

En tout cas, il ne paraît pas logique, sur le plan botanique, de scinder le genre Vitis en 2 genres différents correspondant aux sous-genres précédents. Tout au plus faudrait-il admettre une forte influence du milieu sur la discrimination des génotypes d'un même genre, fait observé par THODAY (1972) au sujet de la sélection disruptive, et des interactions "génotypes $\times$ milieux " en général.

\section{PRISE EN COMPTE DES EFFETS ÉPISTATIQUES DANS LES STRATÉGIES DE SÉLECTION}

A la lumière du modèle génique proposé, il est intéressant d'essayer, d'une part, de généraliser une telle structure héréditaire à d'autres éléments du génome et, d'autre part, de jeter les bases d'une méthode de prise en compte systématique des effets épistatiques en sélection.

Le point central est que l'apparition dans un génome d'un ou plusieurs allèles à effets épistatiques, par croisement voire par mutation, provoque des modifications de ce génome non pas à caractère additif mais à caractère multiplicatif sur tous les loci présentant une mutation modifiable par les allèles introduits. Ce mécanisme, d'ailleurs envisageable au niveau de l'évolution brusque des espèces, serait en tout cas de nature à permettre une amélioration très sensible des variétés avec un ou quelques croisements combinant les gènes à effets épistatiques intéressants avec les mutations à estomper, mais aussi à expliquer un certain plafonnement après les gros progrès génétiques obtenus dans les croisements précédents. Les améliorations possibles, après l'introduction des allèles épistatiques intéressants, seraient surtout lc fait de l'accumulation de mutations valables, indépendamment pour chaque locus. Ce dernier fait justifie la poursuite de la sélection d'une large population de base sur plusieurs cycles dans un schéma récurrent (GALLAIS, 1977) et l'intervention essentielle de l'aptitude générale à la combinaison, alors que la maîtrise des effets épistatiques fait appel surtout à l'aptitude spécifique à la combinaison et au choix judicieux, par des études de disjonction comme chez la vigne ou peut être par voie biochimique, des géniteurs possédant les allèles épistatiques désirés.

Cette démarche pourrait être envisagée de façon plus systématique au départ des programmes de croisements de façon à diminuer les risques de perte de tels allèles chez les premiers descendants. Ceci conduirait à la sélection de lignées possédant les allèles épistatiques identifiés à l'état homozygote et servant de testeurs aux variétés à améliorer, sans pour cela que la méthode pêche par lourdeur excessive puisqu'il serait toujours possible de privilégier certains effets épistatiques très larges sur les caractères clefs de la sélection.

Toute cette réflexion peut être mise en défaut par l'existence, dans le cas de gènes suppresseurs, de certains phénomènes de protection du génome vis-à-vis de ces suppresseurs, ces derniers étant potentiellement capables de bouleverser le génome en question. Mais, en tout état de cause, il est intéressant en génétique quantitative de tenir compte des effets épistatiques qui sont très souvent systématiquement négligés et de rechercher les génotypes possédant ces derniers, puisque l'orientation de la stratégie générale de la sélection en dépend, ce qui est particulièrement important chez les plantes pérennes dont les cycles de croisements ont une durée élevée. Afin de concrétiser cette idée, quelques exemples théoriques simples sont intéressants à exposer. Dans chaque cas il apparaît des différences d'interprétation entre les modèles classiques et ceux faisant intervenir des effets épistatiques:

L'autofécondation du génotype présentant le couple allélique ( $\mathrm{A}, \mathrm{a})$ fournit la disjonction classique : 0,25 AA $+0,5 \mathrm{Aa}+0,25$ aa (donc les $3 / 4$ de phénotype (A). Si « a est une mutation pour laquelle il existe un gène à effet épistatique $(S, s)$ supprimant l'effet de «a», le génotype initial présentant le phénotype $A$ peut être aussi par exemple « a/a, S/s ». Son autofécondation donne la disjonction : $0,25(\mathrm{aa}, \mathrm{SS})+0,5(\mathrm{aa}, \mathrm{S} / \mathrm{s})+0,25(\mathrm{aa}, \mathrm{ss})$, et également les $3 / 4$ de phénotype " $A$ ». Mais, pour ce même résultat phénotypique en $1^{\text {re }}$ génération d'autofécondation, les conséquences des croisements ultérieurs avec d'autres génotypes possédant par exemple l'allèle « $A$ " seront particulières à chacun des 2 cas et donc source de variabilité différente, puisqu'ils se distinguent à la fois pour les allèles de structure et pour les possibilités de recombinaison avec un autre locus, $(S, s)$ en l'occurrence, ce dernier pouvant intéresser d'autres loci.

La prise en compte systématique d'effets épistatiques permet donc d'éclairer de façon nouvelle les stratégies de croisements à partir de résultats préliminaires. Pour chacun de ceux-ci, il est théoriquement possible de trouver des associations "allèles-allèles épistatiques " capables d'expliquer les disjonctions observées. Ensuite, il est utile de confronter les résultats que pourraient procurer le modèle à base d'effets épistatiques facc au modèle classique, eu égard aux croisements ultérieurs réalisables à partir des descendants dans les $1^{\text {res }}$ disjonctions. L'expérience seule, dans des cas précis, renseignera sur la validité générale ou particulière de concevoir un caractère ponctuel comme dépendant non pas d'une seule série allélique mais d'un effet dualiste entre cette série et des gènes à effets épistatiques à prendre très tôt en compte dans un programme de croisements. Pour les caractères floraux chez la vigne, cette idće paraît prévaloir.

Reçu le 2 août 1982. Accepté le 4 mars 1983.

\section{REMERCIEMENTS}

Nous remercions M. LABARRÈre (Université de Bordeaux II) pour les intéressants commentaires qu'il a apportés au sujet de la présentation des diverses explications génétiques du modèle proposé. 


\section{RÉFÉRENCES BIBLIOGRAPHIQUES}

Britten R. J., Davidson E. H., 1969. Gene regulation for higher cells : a theory. Science, $165,349-357$.

Bouquet A., 1978. La Muscadine (Vitis rotundifolia Michx) et sa culture aux Etats-Unis. Connaiss. Vigne Vin, $12(1), 1-20$.

Carbonneau A., 1983. Stérilités mâle et femelle dans le genre Vitis. I. Modélisation de leur hérédité. Agronomie, 3 (7).

Gallais A., 1977. Amélioration des populations, méthodes de sélection et création de variétés. I. Synthèse sur les problèmes généraux et sur les bases théoriques pour la sélection récurrente intra-population. Ann. Amélior. Plantes, 27 (3), 281-329.

Levadoux L., 1956. Les populations sauvages et cultivées de Vitis vinifera L. Ann. Amélior. Plantes, 1, 59-118.

Lewis K. R., John B., 1968. The chromosomal basis of sex determination. Intern. Rev. Cytol., 23, 277-379.
Loomis N. H., Williams C. F., 1957. A new genetic flower type of the Muscadine grape. J. Hered., 48, 294-304.

Rajasekaran K., Mullins M. G., 1979. Embryos and plantlets from cultured anthers of hybrid grapevine. J. exp. Bot., 30 (116), 399407.

Rives M., 1962. Centre d'origine et diversification spécifique dans le genre Vitis. III Congrès Eucarpia, 197-201.

Rives M., 1974. Les vignes sauvages comme sources de gènes pour l'amélioration. Vitis, 13, 186-197.

Rives M., 1975. Les origines de la vigne. La Recherche, 53, 120-129.

Thoday J. M., 1972. Disruptive selection. Proc. R. Soc., Ser. B (London), 182, 109-143. 